\title{
Multi channel EOG Signal Recognition for an Embedded Eye Movement Tracking Device
}

\author{
Matej Kirbiš \\ ASTRON d.o.o. \\ RND Company \\ Jadranska cesta 27, SI-2000 Maribor, Slovenia \\ matej.kirbis@uni-mb.si
}

\author{
Iztok Kramberger \\ University of Maribor \\ Faculty of Electrical Engineering and Computer Science \\ Laboratory for Digital and Information Systems \\ Smetanova ulica 17, SI-2000 Maribor, Slovenia \\ iztok.kramberger@uni-mb.si
}

\begin{abstract}
In this paper we describe a low cost embedded system for eye movement tracking that supports disabled persons with basic interaction with Bluetooth enabled electronic devices. The method is based on tracking the EOG potential. The device has a built-in 32-bit RISC processor that performs acquisition of multichannel differential EOG signals and signal recognition. Signal recognition is based on cross correlation principle where a simplified triangulated linear model for eye movement is used as a reference signal. The eye movement recognition is more or less user independent, but it takes some time and practice to become familiar with the system for efficient use. The embedded eye movement tracking device communicates with other devices via personal network, which is based on Bluetooth wireless technology. The communication protocol at the application level has been simplified in order to gain plain integration with other devices.
\end{abstract}

Keywords; electrooculogram, signal analysis, eye movements, pattern recognition, correlation, microcontroller

\section{INTRODUCTION}

Many diseases and different injuries can lead to a lower level of patient's mobility. As an example, large brain strokes, dystrophy, multiple sclerosis, and injuries to spinal cord can be a reason for high degradation of person's mobility. Though they are of a different origin, they all can cause almost total immobility of the affected person. This condition incapacitates those persons to be able to use common applications and devices in everyday life within the modern information society [1]. Beside this, some of them can communicate only by the eye movements.

Primary goal of our research work is to support these patients with a low cost human machine interface system through which they could communicate with the rest of the world. In this way we can regenerate their use of computers and electronic devices, and consequently their electronic communication with others. Such a device makes it possible for them to enroll in society at least to a certain level and help them to utilize their confined options to the highest limit.

There are many possibilities how to measure eye movements: by camera, special contact lenses and Electrooculogram EOG potential $[2,3]$. This paper presents the possibility to make use of EOG signal.

\section{HARDWARE}

EOG potential is the resting potential of retina. This resting potential sums up between $10 \mathrm{mV}$ to $30 \mathrm{mV}$, and is linear proportional to eye movements in the area between -30 and +30 degrees.

Those signals can be measured by proper electrode attachment. In our case we use three pairs of hypodermic electrodes, that represent three differential EOG signals. (See Figure 1).



Figure 1. Positions of EOG electrodes.

The eye movement tracking device is constructed of three units, analog, digital, and the power unit. At first the differential EOG signals are amplified by a differential multi channel amplifier and then converted to digital signals by an analog to digital converter A/D. (see Figure 2).

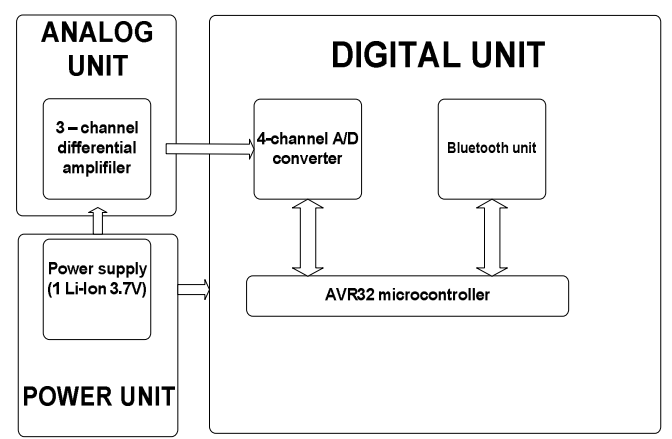

Figure 2. Multi channel embedded device for EOG signal acqusition and processing. 
The analog part consists of four two-stage amplifiers and low pas filters. First stage of each channel amplifier is constructed as a differential amplifier with gain set to 100 . The second stage of each channel amplifier also contains an amplifier with gain set to 10 , and a $50 \mathrm{~Hz}$ low pas filter has been added to this stage. Thus the whole amplifier for each of the channels has amplification of 1000 and highest frequency of the signals is set to $50 \mathrm{~Hz}$ as the acquisition sample rate for each particular channel is $100 \mathrm{~Hz}$.

The electrode A (see Figure 1) represents the common return signal, which is used to compensate the common potential on the user's face and to remove the common mode signals that appear on differential inputs of electrode pairs of each channel as interference signals. The common return signal is formed as an inverted sum of common mode signals from all differential amplifiers in analog fashion with an integration time constant of 2.2 seconds. Common mode rejection factor over $80 \mathrm{~dB}$ is reached, which is almost adequate to 14-bit resolution.

Four of such differential amplifiers amplify the differential EOG signals from four pairs of electrodes, while at the next stage they are acquired and converted into digital form by a four channel 14-bit A/D converter, which is driven by 32-bit RISC AVR32 microcontroller. The main tasks of the microcontroller is to control the $\mathrm{A} / \mathrm{D}$ converter, to sample and buffer the four digital EOG signals, to recognize multi-channel digital EOG signal patterns, and to communicate with other devices over the personal area network PAN. (see Figure 3)
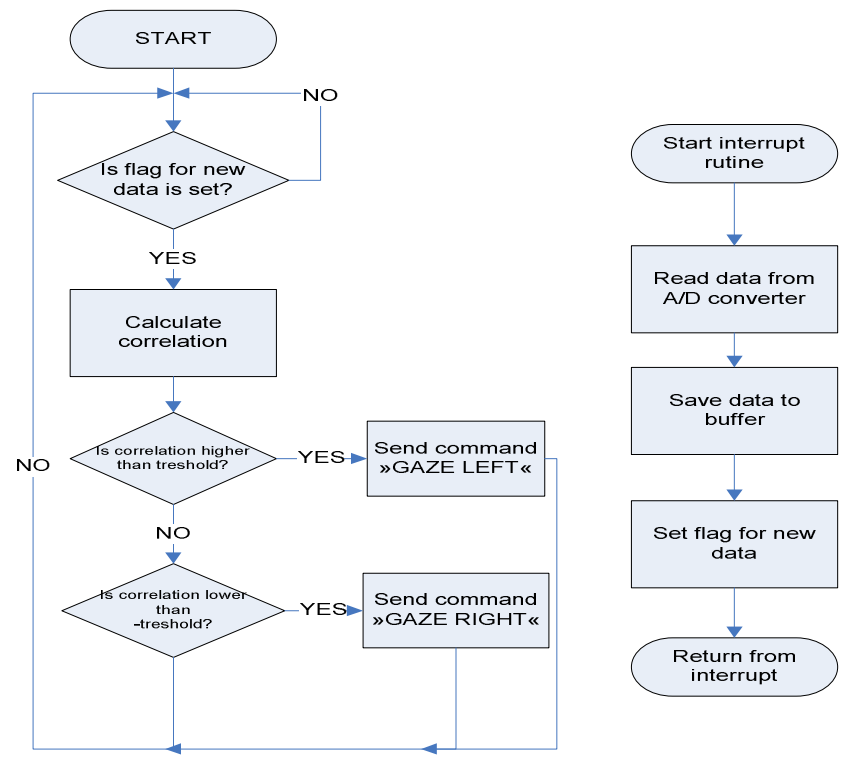

a: main rutine

b: interrupt rutine

Figure 3. Flowchart of the embedded software.

The PAN communication is based on wireless Bluetooth technology, which makes it possible to communicate with Bluetooth enabled devices like personal and portable computers, mobile phones, personal digital assistants PDA, and similar. At the application layer the communication protocol is very simple since it contains only a couple of commands (left, right, up, down, left blink, and right blink) to feature simplified navigation and control within specialized applications that run on the devices connected via Bluetooth [4].

The results of our research work show that the amplitude of the differential analog EOG signals typically varies between $50 \mathrm{uV}$ to $3500 \mathrm{uV}$. By use of the constructed differential amplifier with gain set to 1000 the input signals at the $A / D$ converter inputs consequently vary typically between $50 \mathrm{mV}$ to $3.5 \mathrm{~V}$ (see Figure 4).



Figure 4. Typical EOG signal at the A/D converter input.

\section{Signal RECOGNITION}

Many ways to solve this problem have already been proposed. One of the most known methods is to use signal normalization method, where authors propose normalization of all signals and compare them to already known waveforms. Some of the proposed and published methods represent more advance signal processing algorithms, which involve wavelets [5] [7] and neural networks [6].

The recognition process within our research work represents the implementation of a mapping algorithm that translates multi-channel EOG signals into a simplified command set for the human machine interface. Due to already mentioned low frequency range of the EOG signals we have chosen the correlation principle as one of the most basic approaches to recognize the signal patterns. We have used the cross correlation method that has been defined by equation (1):

$r_{x y}(l)=\sum_{n=-\infty}^{\infty} x(n) y(n-1) \quad 1=0,1,2,3,4$

$\rho_{x x}(l)=\frac{r_{x y}(l)}{\sqrt{r_{x x}(0) r_{y y}(0)}}$

where $\mathrm{x}$ represents the acquired digital input signal, previously windowed by a rectangular window of 100 sample and $y$ represents the reference signal. Windowing to the digital EOG input signals is applied sample wise by moving the window 
for one sample and consequently calculating the cross correlation with the reference signal for each sample.

Values of $\mathrm{r}(\mathrm{x}, \mathrm{y})$ are normalized to the interval $[1,-1]$ by the implication of the equation (2). If the acquired digital input signal and reference signal are completely equal the cross correlation value reaches 1 , and respectively if the calculated cross correlation value drops to -1 this denotes that an inverted or reversed phase signal is present. The case in which the cross correlation value reaches 1 describes a situation when a person gazes into the direction that is adequate to the reference signal, and contrary when the person gazes into opposite direction the cross correlation value reaches -1 . Figure 5 demonstrates the results of the cross correlation (C) with a reference signal (A) and the acquired differential EOG signal (B) from one of the experiments where a person first looks left and then right.
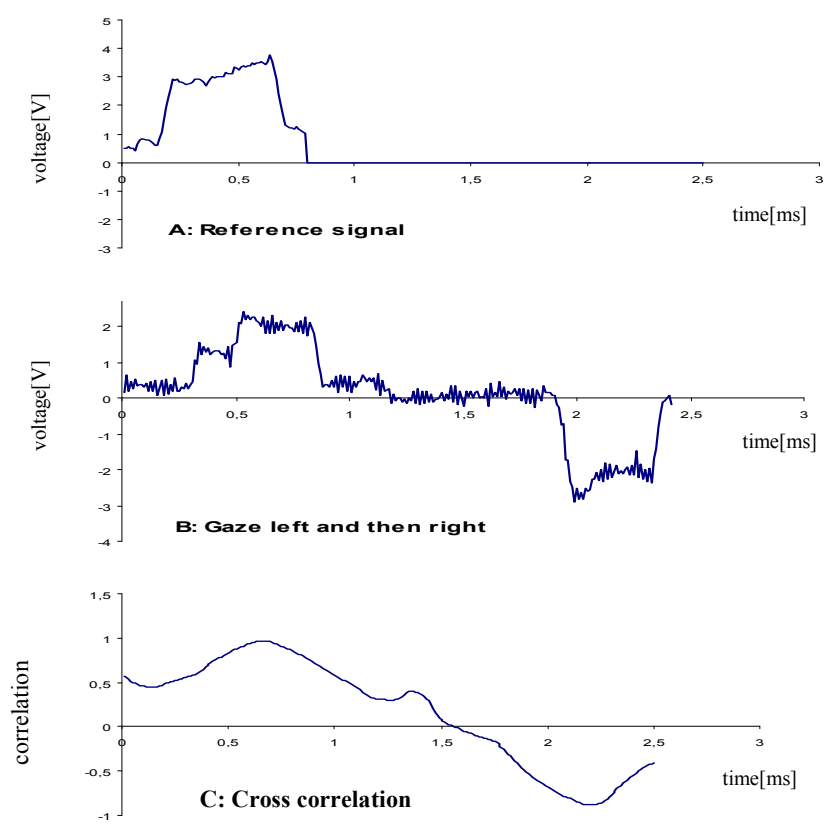

Figure 5. Cross correlation (C) between reference signal (A) and gaze leftright signal (B).

It is shown that the cross correlation value increases when the left gaze input signal is present, and it drops when the right gaze signal is present respectively [8].

Due to the very slow integration constant of the common return signal the DC component appears in the differential input signal. The DC component influences the efficiency of the cross correlation result and therefore it has to be removed from the differential signal. In order to achieve this, we have applied a $1 \mathrm{~Hz}$ first order high pass IIR filter to all the digital input EOG signals at the software layer. The IIR filter is defined by equation (3):

$$
\mathrm{y}(\mathrm{n})=\mathrm{a} 0 \cdot \mathrm{x}(\mathrm{n})+\mathrm{y}(\mathrm{n}-1)
$$

where $x(n)$ and $y(n)$ are input and output signals, and $a 0$ represent first filter pole. The result of the applied high pass filter can be seen on figure 6 , where it can be seen that the DC component has been successfully eliminated, but the shape of resulting EOG signal has also been changed. According to this filtered differential EOG signal the reference signal was defined by a triangulated linear model. The show results are been obtained by a prototype device.

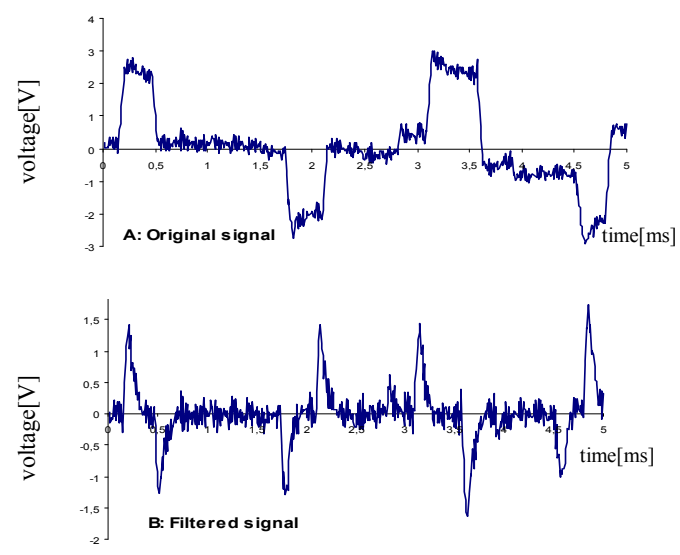

Figure 6. Using first order high pass IIR filter.

The selection and modeling of reference signal is highly important as it has a direct influence on the recognition process efficiency. One option would be to use a previously captured part of the input signal as the reference signal (A), but we decided to use a simplified triangulated linear model as the reference signal (B), which was approximated from the original input signal (A) (see figure 7).
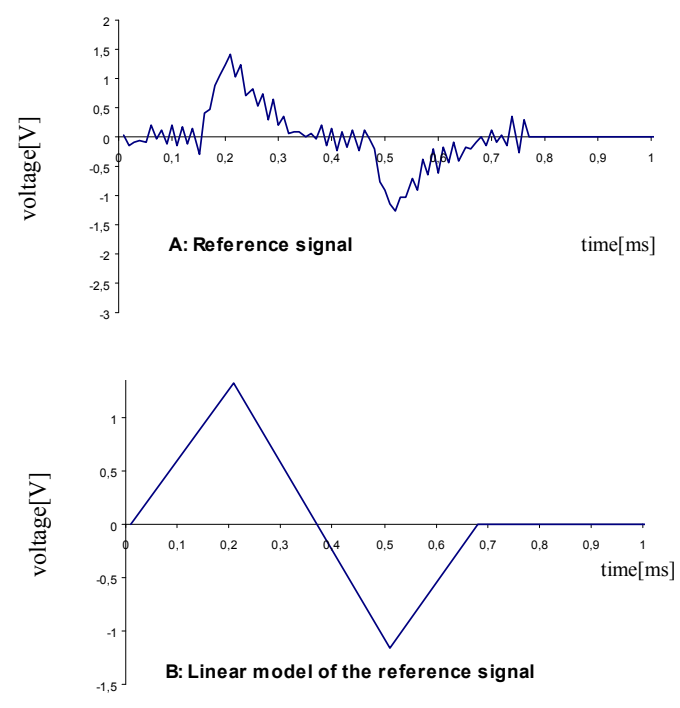

Figure 7. Captured part of the input EOG signal (A), and its appurtenant simplified triangulated linear model (B).

The simplified triangular model was used in order to achieve better adaptation to different amplitudes and timings of the input signals. By use of the proposed simplified triangular model it is understandable that the signal pattern recognition process requires an appropriate threshold value in order to successfully recognize eye movements. 


\section{RESUlts}

The following example shows an acquired differential EOG signal between $G$ and $F$ electrodes (see figure 1). The input EOG signal (B) shown on the figure 7 is constituted of five gazes to the left and four gazes to the right. In the first case a part of a previously captured input signal was used as the reference signal (A), and the DC component was not removed from the input signal.
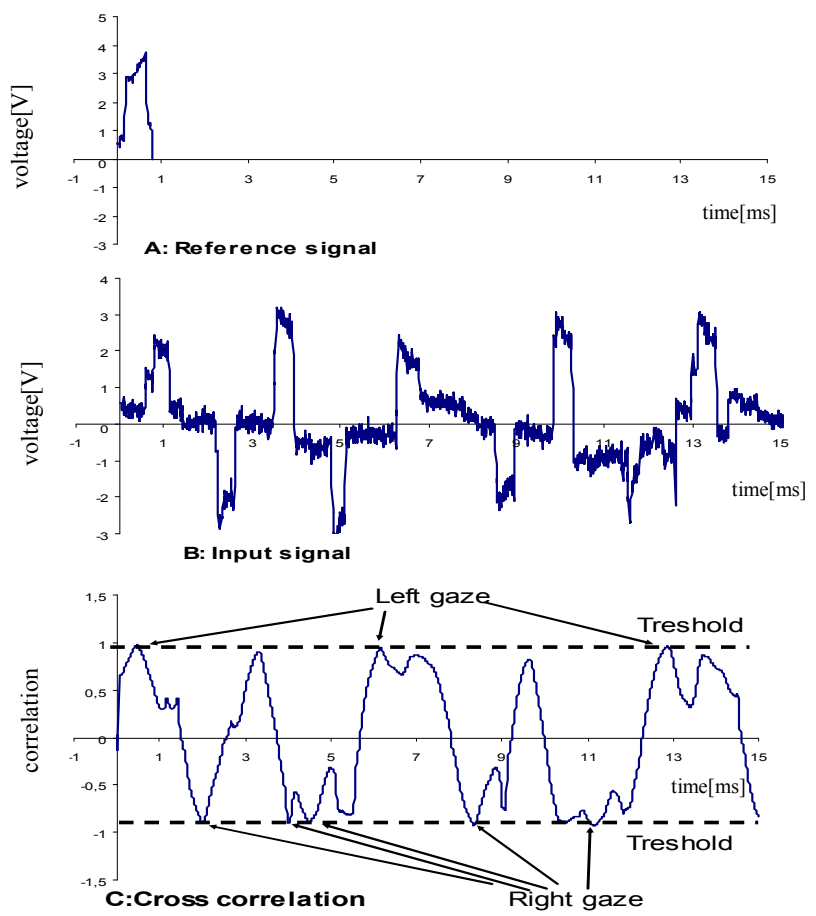

Figure 8. Left and right gaze recognition results on the original input EOG signal with a previously captured reference signal.

In such a case it is difficult to define the appropriate threshold value as the DC component influences the computation of the cross correlation value $(\mathrm{C})$ and drops the recognition rate.

The EOG input signal (B) for the next example (see figure 8) is the same as within the previous example, but the high pass filter was applied to remove the DC component. The simplified triangulated linear model (A) was used for the reference signal. In this case the resulting cross correlation function shows better recognition results as we eliminated the influence of the DC component within the input signal and consequently it is easier to define the appropriate threshold value.

\section{CONCLUSION}

The implementation of an embedded battery powered low cost device is shown, which includes the EOG signal pattern recognition algorithm for eye movement tracking in order to support disabled people with mappings of recognized eye gaze gestures to a simplified command set for navigation and control of Bluetooth enabled devices. Because of the limited processing power the cross correlation principle was used as the recognition method, which proved it self to be suitable for this purpose with use of a simplified triangulated linear model for the reference signal. The shown results have been obtained by a prototyped device.
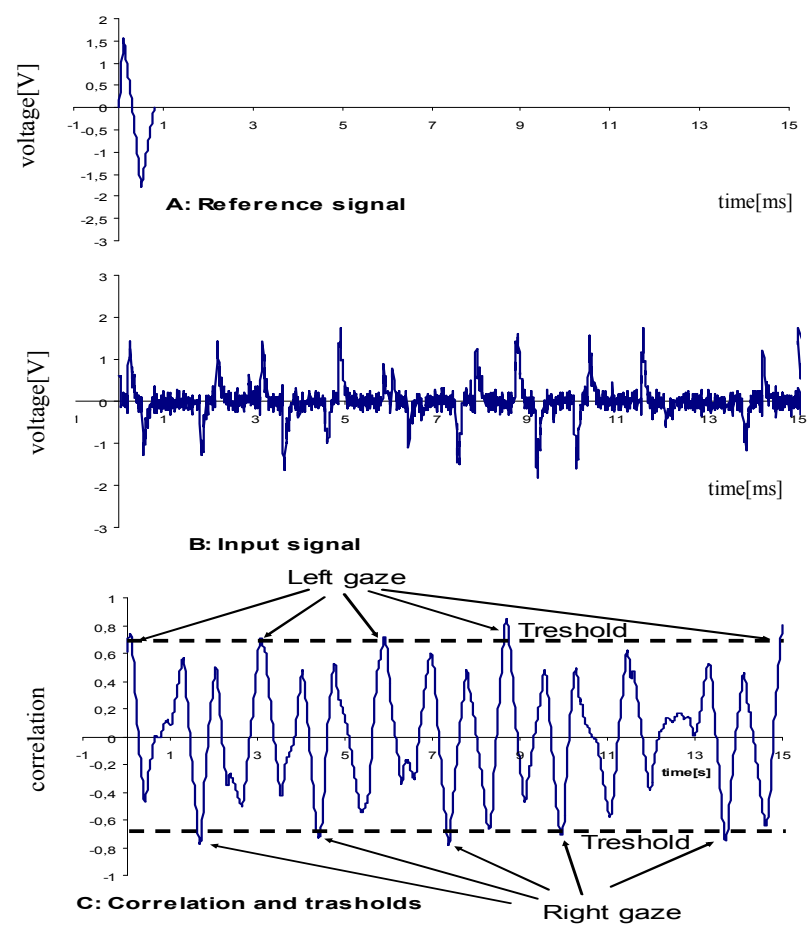

Figure 9. Left and right gaze recognition results with use of simplified triangulated linear model as the reference signal, and high pass filter applied to the input EOG signal.

\section{REFERENCES}

[1] J. Gips, Olivieri, "Eagle Eyes: An Eye Control System for Persons with Disabilites," 11 th Internetional Conference on technology an Pesons with Disabilities, Los Anglese, 1996.

[2] R. Barea, L. Boquete,M. Mazo, E. Lopez, "System for Assisted Mobility Using Eye Movements Based on Electrooculography," IEEE Transaction on Neual Systems and Rehabilitation Engineering, vol. 10, no. 4, pp. 209-218, December 2002.

[3] D. Kumar, E. Poole, "Classification of EOG for Human Computer Interface," Proceedings of the Second Joint EMBS/BMES Conference Houston, TX. USA, 2002.

[4] D. Qiuping, T. Kaiyu, Li Guang, "Development of an EOG (ElectroOculography) Based Human-Computer Interface," in Proc. of 27th Annual Conference on IEEE Engineering in Medicine and Biology, Shanghai, China, 2005.

[5] S. Mallat, "A Wavelet Tour of Signal Processing," CA Academic, SanDiego, 1998.

[6] R. Barea, L. Boquete, M. Mazo, E. Lpoez, L. M. Bergasa, "E.O.G.guidance of wheelchair using neural networks," Proc. 15th Int. Conf. onPattern Recognition, Barcelona, 2000.

[7] S.Durand, J.Froment, "Artfact Free Signal Processing with Wavelets," IEEE Intr. Conf on Acoustics, Speech and Signal Proc., ICASSP, 2001.

[8] S.K. Kang, Y.U. Kim, I.M. So, S.S. Lee, S.T. Jung, "EOG and Marker Recognition for Wearable UserInterface," Dept. of Computer Engineering. 